\title{
CONSUMO DE ALCOHOL Y OTRAS DROGAS EN EL MEDIO LABORAL
}

\author{
CONSUMPTION OF ALCOHOL AND OTHER DRUGS IN THE \\ OCCUPATIONAL ENVIRONMENT
}

\author{
ENRIQUETA OCHOA MANGADO ${ }^{(1)}$, AGUSTÍN MADOZ GÚRPIDE(2)
}

(1) Servicio de Psiquiatría. Hospital Universitario Ramón y Cajal. Madrid. España.

(2) Centro de Salud Mental de San Blas. Madrid. España.

\section{RESUMEN}

El consumo de alcohol y otras drogas tiene una elevada prevalencia en la sociedad en general, y también entre la población trabajadora, repercutiendo sobre el medio laboral. La repercusión del consumo de estas sustancias en el medio laboral es muy importante (enfermedades, accidentes laborales, absentismo, incapacidades laborales, disminución de la productividad...). Se valora la necesidad de una política en el medio laboral encaminada a prevenir o minimizar los riesgos laborales derivados del consumo de estas sustancias, con programas de prevención y apoyo que aporten información básica de referencia y orienten al abordaje asistencial de los trabajadores afectados.

Palabras clave: Alcohol, droga ilegal, ámbito laboral, prevención, legislación laboral.

(Med Segur Trab 2008; 54 (213):25-32)

\begin{abstract}
The consumption of alcohol and other drugs has high prevalencia in the society in general, and in the population of workers especially, affecting on the occupational area. The repercussion of the consumption of these substances in the working enviroment is very important (diseases, occupational accidents, absenteeism, occupational disabilities, decrease of the productivity...). They are necessary political measurements in the occupational area to prevent and to minimize the risks derived from the consumption of these substances. There should be included programs of prevention and support, which offer basic information and orientation to the medical aproach of the affected workers.
\end{abstract}

Key words: Alcohol, illegal drug, occupational area, prevention, occupational legislation.

(Med Segur Trab 2008; 54 (213):25-32)

\footnotetext{
Correspondencia:

Dra. Enriqueta Ochoa Mangado

Servicio de Psiquiatría.

Hospital Universitario Ramón y Cajal.

Ctra Colmenar Km 9,100.

28034 Madrid (España)

eochoa.hrc@salud.madrid.org
} 


\section{INTRODUCCIÓN}

La legislación sobre materia laboral en España surge dentro del marco de la Constitución ${ }^{1}$ que indica el deber de los poderes públicos por velar por la seguridad e higiene en el trabajo (Art. 40), y el derecho a disfrutar de un medio ambiente laboral adecuado para el desarrollo de la persona, así como el deber de conservarlo (Art. 45). Estos deberes y derechos plantean la necesidad de desarrollar políticas de promoción de la salud en el trabajo. Dentro de estas políticas se encuentran las de prevención y asistencia de problemas relacionados con el alcohol y otras drogas, que se enmarcan también en el marco de las leyes sobre Salud y Seguridad en el Trabajo.

Las condiciones de trabajo son una parte importante en la vida y pueden tener una decisiva influencia en los niveles de salud y bienestar, dentro y fuera del ámbito laboral. La prevención de riesgos laborales es un deber de las empresas y un derecho de los trabajadores, los cuales también deben velar por la protección de su propia salud y la de sus compañeros. La Unión Europea considera un objetivo básico de las políticas laborales la protección de la salud de los trabajadores mediante la prevención de riesgos directamente derivados del trabajo y de los riesgos que pueden afectar al rendimiento laboral.

La gravedad de los consumos de alcohol y/o otras drogas en el medio laboral ha sido reconocida desde hace tiempo por la Organización Internacional del Trabajo, que considera que el consumo de alcohol y de drogas es un problema que concierne a un número elevado de trabajadores ${ }^{2}$. Señala que los efectos nocivos del consumo de alcohol y drogas en el lugar de trabajo y en el rendimiento laboral son corrientes a todos los niveles. La seguridad profesional y la productividad pueden verse afectados de forma adversa por empleados bajo el influjo del alcohol o las drogas $^{3}$. Indica también como el consumo de alcohol y drogas por los trabajadores produce un fuerte impacto, que se extiende a familia y compañeros de trabajo que son afectados en términos de pérdida de ingresos, estrés y baja moral ${ }^{4}$. Por tanto el consumo de alcohol y otras drogas es un problema que no sólo afecta al consumidor, sino también al ambiente laboral y la eficacia de una empresa.

\section{PREVALENCIA DE CONSUMO DE ALCOHOL Y OTRAS DROGAS EN EL MEDIO LABORAL}

La elevada prevalencia entre la población trabajadora del consumo de alcohol y/o otras drogas con- vierte estos consumos en un problema de gran magnitud. Los primeros estudios a nivel nacional sobre el consumo de drogas en el medio laboral ${ }^{5,6}$ así como estudios en Estados Unidos ${ }^{7}$ y Europa ${ }^{8}$ corroboran las elevadas prevalencias, con cifras semejantes a las referidas por las Encuestas Domiciliarias sobre Consumo de Drogas 9 , las Encuestas sobre Drogas a la Población Escolar ${ }^{10}$ y el Observatorio Español sobre Drogas ${ }^{11}$.

Respecto al alcohol, según la última Encuesta Domiciliaria sobre Consumo de Drogas ${ }^{9}$, su consumo y abuso alcanza prevalencias más altas en la población activa asalariada que en el total de la población. Un $95 \%$ de la población laboral ha tomado bebidas alcohólicas alguna vez y el $90 \%$ lo han hecho en los últimos doce meses. Estiman que el $12 \%$ de los trabajadores beben con niveles de consumo que suponen un riesgo para la salud. El 26\% de los bebedores han presentado problemas derivados por el uso de alcohol, y casi un 7\% de los bebedores han sufrido algún tipo de consecuencia laboral negativa por el uso del alcohol (accidentes, sanciones, absentismo, despidos).

Un reciente estudio realizado en 2006 en la Comunidad de Madrid, encuentra también que el $95 \%$ de los ocupados consumió alcohol alguna vez, el $93 \%$ lo hizo en los últimos doce meses, $86 \%$ en el último mes y $65 \%$ en la última semana. La cantidad media de alcohol consumido es de $45 \mathrm{ml}$./día $(41 \mathrm{ml}$. en días laborables y $48 \mathrm{ml}$. los fines de semana), siendo mayor en varones que en mujeres. Los consumos más altos los realizan la población entre 2434 años los días laborables (46 ml./día de media) y los de 16-24 años en fin de semana (72 ml./día de media). Al valorar la frecuencia de consumo, el 40\% bebió con frecuencia media ( 1 a 4 veces/semana), y el $13 \%$ lo hizo con frecuencia elevada ( 5 a 7 veces/semana). Según la cantidad diaria ingerida, el $23 \%$ son bebedores moderados (21-60 gr./día en varones y $21-40$ gr. en mujeres), y el $10 \%$ son bebedores abusivos (más de 60 gr./día en varones y 40 gr./ día en mujeres). De este $10 \%$, un $2,5 \%$ beben con frecuencia elevada (diario o casi diario) un 5,6\% lo hacen con frecuencia media (hasta cuatro días por semana), y tan sólo un 1,9\% bebían 1 o 2 días en semana. Los bebedores abusivos son con mayor frecuencia hombres $(12,6 \%)$ que mujeres $(6,9 \%)$. Por edad destacan los de 16-24 años $(15,2 \%)$ y los de 25 34 años (10\%). El lugar donde se consume con más frecuencia es en bares, pubs y cafeterías, pero casi el $2 \%$ lo hace en el trabajo ${ }^{12}$.

Otros estudios en nuestro medio encuentran 
Consumo de alcohol y otras drogas en el medio laboral

Enriqueta Ochoa Mangado, Agustín Madoz Gúrpide

cifras más altas, ya que según éstos el $14 \%$ de los trabajadores son bebedores abusivos ${ }^{13,14}$.

El cannabis es la sustancia psicoactiva ilegal más consumida, y entre determinados segmentos de población tiene prevalencias casi tan importantes como el tabaco y el alcohol. Un 54\% de los activos laboralmente refieren haberlo consumido alguna vez, el 34\% lo han hecho en los últimos doce meses y el $27 \%$ en los últimos treinta días. El $10 \%$ de los trabajadores refiere su consumo habitual (de 3 a 7 veces/semana). Consumen con más frecuencia los varones, y aquellos entre 16-34 años ${ }^{12}$. Estos consumos son mucho más frecuentes que los referidos en estudios más antiguos, que indicaban un consumo en el último mes en población activa del $7-9 \%{ }^{13-15}$.

La cocaína es también una sustancia ilegal bastante consumida entre algunos segmentos de la población laboral. Un $22 \%$ de los activos laboralmente han consumido cocaína alguna vez, un $12 \%$ lo han hecho en los últimos doce meses y el 7,5\% en los últimos treinta días. El consumo es más esporádico que en otras sustancias: así el 0,4\% refiere consumirla con frecuencia diaria o casi-diaria. Los varones consumen más que las mujeres, especialmente los de 16-34 años ${ }^{12}$. Esta prevalencia de consumo ha aumentado de forma importante en los últimos 10 años, que referían prevalencias mensuales entre el 1,6-3\% ${ }^{13-15}$.

El consumo de alucinógenos, drogas de síntesis y anfetaminas está muy asociado entre ellas, y también al de cocaína y cannabis. Aunque su uso frecuente no es muy amplio, aunque en ciertos segmentos de población laboral como entre los varones de 16-24 años es importante, con una prevalencia del 7-10\% en los últimos doce meses ${ }^{12}$. Este consumo ha aumentado en los últimos años, pasando de 0,8-1\% hace 10 años al $1-3 \%$ actual $^{13-15}$.

Un $0,4 \%$ de los activos laboralmente han consumido heroína alguna vez y un $0,1 \%$ lo hicieron en los últimos doce meses. Parece que el consumo de heroína en población laboral ha descendido claramente en los últimos 10 años. El perfil de sus consumidores en situación laboral activa corresponde a varones (3/1), de edades entre 25-34 años actual $^{12-15}$.

Entre los consumidores de drogas ilegales, el $33 \%$ ha experimentado alguna consecuencia negativa y un $7,6 \%$ presentó consecuencias laborales negativas $^{14}$.

\section{CONSECUENCIAS DERIVADAS DEL CONSUMO DE DROGAS}

El 55,7\% de los trabajadores consideran que el alcohol y las drogas son un problema importante en el mundo laboral. Enfermedades, accidentes laborales, disminución de la productividad y mal ambiente entre compañeros, son las principales razones por las que el alcohol y las otras drogas se consideran un problema en el mundo laboral ${ }^{12}$.

La repercusión que el consumo de alcohol y/o otras drogas produce en el medio laboral es muy importante. Según estudios de la International Labour Organization ${ }^{16}$, los trabajadores que consumen alcohol y otras drogas presentan un absentismo laboral de 2 a 3 veces mayor que el resto de los trabajadores. La bajas por enfermedad de estos trabajadores dependientes del alcohol u otras drogas tiene un coste 3 veces mayor a las del resto de los empleados, a la vez que esas mismas personas perciben también indemnizaciones por un importe 5 veces mayor que el resto de sus compañeros. El 20-25\% de los accidentes laborales ocurren en trabajadores que se encuentran bajo los efectos del alcohol u otras drogas, y causan lesiones a ellos mismos u a otros. El consumo de alcohol y otras drogas afecta negativamente a la productividad, ocasionando importantes perjuicios a empresarios y trabajadores por absentismo, desajustes laborales, incapacidades laborales, rotación del personal, reducción del rendimiento laboral y mala calidad del trabajo ${ }^{17}$.

En España se calcula que entre el $15-25 \%$ de la siniestrabilidad laboral se debe a problemas relacionados con el alcohol. Los bebedores tienen 3 veces más accidentes en el trabajo que otros trabajadores, perdiendo muchos más días laborables por enfermedad. El uso de cannabis afecta negativamente a la capacidad de realizar tareas que requieren atención y concentración, aumentando el riesgo de accidentes laborales. Muchos consumidores de cocaína pueden tener problemas, tanto en tareas que requieran atención y concentración como por absentismo laboral y otras consecuencias. El uso de estimulantes incrementa el riesgo de padecer accidentes de trabajo o de tráfico. Estudios nacionales en población laboral mostraron que el $23-31 \%$ de los trabajadores consumidores de drogas han tenido alguna consecuencia negativa derivada de sus consumos, y el 13-15\% fueron de carácter laboral (bajas laborales, absentismo, accidentes laborales, tensiones laborales, bajo rendimiento laboral...) ${ }^{12}$. 


\section{FACTORES DE RIESGO EN EL CONSUMO DE ALCOHOL Y OTRAS DROGAS}

La causalidad del uso de drogas es multifactorial, influyendo factores personales, familiares y sociales, como la búsqueda de satisfacción inmediata o la tendencia a la evasión y al aturdimiento ante determinadas situaciones ${ }^{18}$. Factores no laborables y laborables pueden influir el consumo de alcohol, cocaína, y cannabis.

Diversas variables se consideran factores de riesgo no laborales, ya sean familiares, sociales o personales (Tabla I).

\section{Tabla I: Factores de Riesgo no Laborales}

- Consumo de alcohol abusivo o drogas por los padres

- Consumo de drogas en lugares de diversión

- Malas relaciones fraterno-filiales

- Conocer a consumidores de drogas

- Facilidad para obtenerlas

- Sentirse agobiado y en tensión

- Pérdida de confianza en sí mismo

- No participación social

Los factores laborales que se consideran de riesgo para el consumo se describen en la Tabla II.

\section{Tabla II: Factores de Riesgo Laborales}

- Trabajos a destajo y alto rendimiento

- Tareas rutinarias y monótonas

- Insatisfacción en el trabajo

- Jornadas de trabajo prolongadas

- Turnos cambiantes

- Largas ausencias de casa

- Cansancio intenso en el trabajo

- Compañeros consumidores

- Trabajos dependientes de la relación social

- Disponibilidad en el medio laboral

Estos factores laborales pueden tener un peso real superior al explicitado verbalmente entre las razones para consumir alcohol u otras drogas ${ }^{18}$.
Además, el entorno laboral puede comportar la exposición a otros factores de riesgo para el abuso de drogas, aumentando la probabilidad de desarrollar problemas derivados del consumo de drogas. Hay trabajos que potencian situaciones de riesgo alcohólico u de otras drogas, como aquellos en los que hay disponibilidad de alcohol (bares, etc), presión social para beber o consumir estimulantes en trabajos dependientes de la relación social, o presión del entorno laboral donde otros trabajadores consumen habitualmente.

En relación con la presencia de las drogas en el lugar de trabajo, el 55\% indica que conoce a algún compañero de trabajo que bebe en exceso o consume alguna droga ${ }^{12}$. Entre los trabajadores, los más jóvenes (de 16 a 34 años) y especialmente los de 12 a 24 años, son los que están en situación de mayor riesgo. Estos jóvenes son los que tienen prevalencias más altas de abuso de alcohol y también de consumo de cannabis, éxtasis o cocaína. Y por estos consumos pueden presentar consecuencias negativas, tanto a nivel de salud, familiar, social y laboral. Por esta situación de mayor riesgo y vulnerabilidad, en especial los de 16 a 24 años, se requiere acciones prioritarias en las empresas y por parte de las instituciones. Estas acciones se basarán en una buena información sobre los riesgos de las drogas, formación en actitudes saludables, prevención en ciertos ámbitos (familia, ocio, relaciones grupales...), así como una pronta atención cuando empiezan a sufrir las primeras consecuencias de los consumos.

\section{POLÍTICAS DE ACTUACIÓN}

La importancia de actuar en el campo de las adicciones deriva de los enormes costes directos e indirectos que el consumo de alcohol, cocaína u otros psicoestimulantes, cannabis, heroína, psicofármacos... provocan en el medio laboral, al generar problemas de salud, incrementar los riesgos de accidentes laborales, favorecer la conflictividad, las bajas laborales, el absentismo, la disminución de la productividad... ${ }^{19}$.

Las administraciones sanitarias, en el marco de sus competencias tienen que promover actuaciones que permitan conocer la salud de los trabajadores y posibiliten el desarrollo de programas de prevención para los problemas detectados. Deben de poner en marcha en el medio laboral programas de prevención sobre el consumo de alcohol y otras drogas, así como impulsar programas asistenciales que faciliten la atención de los trabajadores que lo precisen. Los costes derivados del uso de sustancias en el lugar de 
trabajo superan ampliamente los que puedan derivarse de las programas de prevención y asistencia ${ }^{20}$.

Los programas de prevención y apoyo en relación con las drogas deben realizarse en cualquier empre$\mathrm{sa}$, independientemente de su tamaño. Deberán ser flexibles y adaptables a las distintas realidades. Estos programas contarán con la participación de los diversos sectores de la empresa y de los empleados, respondiendo a sus preguntas e inquietudes, y presentándolos de forma positiva, no sancionadora.

Los programas para prevenir o minimizar los problemas de consumo deben abarcar diversos aspectos, que van desde la información hasta la facilitación de la asistencia de los trabajadores enfermos que lo deseen ${ }^{21}$ (Tabla III).

\section{Tabla III: Aportaciones que deben hacer los programas de prevención}

- Información básica de referencia

- Pautas para plantear actuaciones preventivas

- Información sobre recursos

- Orientación para el abordaje asistencial de los trabajadores con problemas

- Criterios de derivación asistencial de los problemas que no puedan atenderse en los recursos laborales

Los programas de prevención encaminados a mejorar la situación de los trabajadores en relación con el consumo de drogas deben promover cambios de estilo de vida, e incluso de cultura de la salud dentro de la empresa. Para ello es importante conocer las actitudes y conductas de los trabajadores hacia los consumos, antes de definir los objetivos de carácter preventivo y asistencial.

Las políticas preventivas en relación con el alcohol y otras drogas deben de ser informativas, de protección y de sensibilización. Con carácter preventivo se facilitará el no consumo en la empresa (supresión de venta interior, mejorar las condiciones de trabajo....). Respecto a los trabajadores se buscará cambiar actitudes y conductas en relación al consumo, con información amplia, clara y ajustada a la realidad sobre las drogas y las consecuencias sobre su abuso ${ }^{21}$.

Se buscará detectar precozmente los problemas existentes relacionados con el consumo y facilitar la atención a los trabajadores adictos que lo deseen.
Los procedimientos diagnósticos para detectar los problemas deben ser conocidos y aceptados voluntariamente por los usuarios, respetando su confidencialidad. Los tratamientos se propondrán evitando la marginación de la persona a tratar y se facilitará la normalización y reinserción laboral.

Dentro de las políticas para controlar el consumo de alcohol en la empresa pueden realizarse diversas medidas como supresión de la venta de alcohol dentro del recinto de la empresa, detección precoz de bebedores excesivos, detección de problemas relacionados con el alcohol, ofertar a los bebedores excesivos y a los dependientes atención personalizada para reducir o abandonar el consumo de alcohol. Si lo acepta, asegurar la confidencialidad y proteger la seguridad del puesto de trabajo. Si no acepta la ayuda y el problema continúa o se agrava, aplicar medidas disciplinarias escalonadas, evitando que sean irreversibles ${ }^{21}$.

En la detección precoz de los consumos puede ser útil prestar atención a diversos síntomas que sin ser patognomónicos orientan a pensar en abuso de alcohol y/o otras sustancias ${ }^{21}$ (Tabla IV).

\section{Tabla IV: Síntomas que orientan al diagnóstico de abuso de sustancias}

- Accidentabilidad reiterada

- Consultas frecuentes por problemas de salud inespecíficos

- Problemas gastrointestinales

- Somnolencia

- Movimientos inestables o temblores

- Pupilas anormalmente dilatadas o contraídas

- Ojos enrojecidos

- Agresividad, irritabilidad o intolerancia crecientes

- Cambios bruscos del humor

- Comportamientos violentos

- Desconfianza excesiva hacia los demás (recelo hacia compañeros de trabajo, evitación de superiores)

- Deterioro de la higiene personal y del vestir

- Hurtos

- Problemas familiares

- Problemas financieros

- Petición frecuente de dinero a los compañeros de trabajo

- Absentismo

- Retrasos en el inicio del horario laboral y salidas anticipadas

- Trabajo de inferior calidad, o errores en el trabajo 
Se debe de valorar la cantidad consumida, el patrón de consumo y si presentan criterios de dependencia de alcohol. El CAGE puede ser útil en la detección de problemas relacionados con el alcohol $^{22}$. En bebedores de riesgo sin dependencia se informará de los riesgos que pueden producir el uso de alcohol y las ventajas de disminuir la ingesta. La realización de controles de orina pueden ser útiles para la determinación reciente de consumo de drogas, pero debe realizarse con las garantías jurídicas y éticas necesarias.

La política de drogas de la empresa debe garantizar que su objetivo no es perseguir a los adictos, sino contribuir a mejorar las condiciones de salud y seguridad en la empresa. El hecho de que la embriaguez y el consumo de drogas sea motivo de sanción o despido para los trabajadores puede dificultar las políticas de drogas de la empresa. En lugar de asumir una posición punitiva y sancionadora, la empresa debe contribuir a prevenir, detectar precozmente o mejorar situaciones problemáticas en relación con las drogas. La legislación vigente regula el despido disciplinario por causas derivadas del consumo de alcohol y otras drogas si repercuten negativamente en el trabajo $^{23}$. Esta medida debe de ser la última a adoptar, y antes de ella deben de utilizarse sanciones intermedias, como faltas leves, graves y muy graves.

La mayor parte de las empresas no han establecido protocolos para la identificación precoz de los trabajadores que presentan problemas con el alcohol u otras drogas. En general, las empresas no reaccionan hasta que se produce una crisis originada por el grave comportamiento del trabajador y existe grave repercusión en el medio laboral. Cuando las circunstancias obligan a la empresa a tomar decisiones, pueden ser excesivas por temor a las repercusiones negativas, con sanciones disciplinarias que con frecuencia excluyen más al dependiente.

Algunos autores recomiendan un protocolo de actuación para los pacientes con trastornos mentales graves, que es aplicable a los pacientes con adicción a alcohol u otras drogas ${ }^{24-26}$.

Confrontar al trabajador enfermo con las alteraciones que padece y sus consecuencias laborales, por parte de las personas más adecuadas como médico de empresa, especialista de prevención de medios laborales, superior jerárquico, miembro del equipo de dirección....

Valorar el grado de conciencia respecto a los consumos que presenta, a sus conductas y a la posible motivación para iniciar tratamiento. La frecuente minimización de los consumos que realiza el adicto debe confrontarse con la repercusión en las conductas que realiza.

Documentar las alteraciones de conducta del trabajador, percibidas por compañeros y/o supervisores, jefes, representantes sindicales u otros.

Comunicar por escrito a la Directiva del centro y al Servicio de Prevención de riesgos laborales, solicitando valoración del trabajador dependiente de sustancias.

Citar al trabajador por vía convencional en el servicio de Prevención, según el art. 22 de la ley $31 / 95^{27}$ para realizar examen del estado de salud, con las garantías habituales de confidencialidad, etc. y se emitirá un juicio clínico-laboral y grado de aptitud para su puesto de trabajo.

Si el trabajador no acude a la citación previa, citar por escrito mediante carta certificada o burofax para reconocimiento médico obligatorio por sospecha de riesgo para él mismo o para terceros, tras la aprobación preceptiva del Comité de Salud y Seguridad del centro. Si tampoco acude se realizará comunicación a Dirección-Gerencia para solicitar valoración médica involuntaria por vía judicial.

Tras la evaluación el trabajador será remitido a su médico de referencia para ser evaluado por los profesionales sanitarios convenientes.

Si el trabajador niega el consumo y las alteraciones conductuales, se consultará con sus familiares si es posible.

Siempre que sea posible, se modificará el trabajo del paciente para hacer lo posible que siga trabajando.

En casos de máxima gravedad, se activarán los protocolos de intervención urgente con intervención de los profesionales que marcan la ley de Seguridad de Prevención de riesgos Laborales.

Aunque el artículo 35.1 de la Constitución ${ }^{1}$ establece que los ciudadanos tienen el derecho y el deber de trabajar, se trata de una capacidad que precisa determinadas aptitudes funcionales para llevarla a la práctica. Por ello además de valorar el diagnóstico y la situación en que se encuentra el adicto, es importante valorar las capacidades del afectado para el empleo específico ${ }^{28}$. Su posibilidad de trabajar 
depende de múltiples variables, como el grado de adicción y si se encuentra en fase de consumo o de abstinencia, la afectación de los consumos en sus funciones ejecutivas, considerando también la adecuada competencia psicosocial que mantenga y las demandas que le provoca un determinado trabajo. Es importante valorar los recursos cognitivos que mantiene y el riesgo de que sus conductas y enfermedad ocasionen malestar clínicamente significativo o deterioro del funcionamiento laboral ${ }^{26}$.

No es infrecuente que durante la intoxicación de alcohol u otras drogas (principalmente estimulantes) se produzcan amenazas o conducta violentas en el medio laboral. Si estas aparecen se utilizará las mismas estrategias de intervención que en las conductas agresivas fuera del marco de la intoxicación ${ }^{26}$ :

Técnica de desactivación o de desescalada. En primer lugar, verbalmente, se tratará de reducir la tensión, dirigiéndose al paciente de forma tranquila y no provocadora, intentando establecer una conversación y plantearle otras alternativas más eficaces, así como ofrecer medicación para su toma voluntaria si lo precisa.

Intervención contra la voluntad del trabajador, con empleo de contención física, sujeción mecánica e intervención farmacológica si existe riesgo elevado para él o para otros.

\section{BIBLIOGRAFÍA}

1. Constitución Española. Título primero. De los derechos y deberes fundamentales. 1978.

2. OIT. Iniciativas en el lugar de trabajo para prevenir y reducir los problemas causados por el alcohol y otras drogas. Ginebra 1988.

3. OIT. Proyecto de repertorio de recomendaciones prácticas sobre el tratamiento en los lugares de trabajo de los problemas que plantea el consumo de alcohol y drogas. Ginebra 1994.

4. OIT. Lucha contra el abuso de alcohol y drogas en el lugar de trabajo. Ginebra 1998.

5. Navarro J. (Coord.) EDIS S.A. Las drogodependencias en el mundo laboral. Dto. Servicios Sociales UGT. Madrid 1987.
Una vez sedado se vigilará de forma continuada y se aplicarán los exámenes médicos precisos para el diagnóstico y tratamiento más eficaz.

\section{CONCLUSIONES}

El consumo de alcohol y/ otras drogas es muy prevalente en el medio laboral. Se estima que el 10\% de los activos laboralmente son bebedores de riesgo (más de $40 \mathrm{gr} . /$ día en mujeres y de 60 gr./día en varones), y el $8 \%$ son bebedores abusivos y frecuentes. Las prevalencias consumo de otras drogas en el último mes se estiman en $27 \%$ para el cannabis, $7,5 \%$ para la cocaína, 2,7\% para las drogas de síntesis y $1 \%$ para los alucinógenos. El consumo de heroína es menor del $0,1 \%$.

La repercusión que el consumo de alcohol y/o otras drogas produce en el medio laboral es muy importante. Producen importantes perjuicios a la empresa y a sus compañeros por el aumento de enfermedades, accidentes laborales, absentismo, e incapacidades laborales, junto con disminución de la productividad, y mal ambiente laboral. Por ello son necesarios programas de prevención y apoyo en relación con el consumo de alcohol y otras drogas en cualquier empresa. Estos programas aportarán información básica de referencia, pautas para plantear actuaciones preventivas, orientación para el abordaje asistencial de los trabajadores con problemas y criterios de derivación asistencial.

6. Navarro J. (Coord.) EDIS S.A. La incidencia de las drogas en el mundo laboral. Fundación de Ayuda contra la Drogadicción. Madrid 1996.

7. NSDA. National ousehold Survey on Drug Abuse: population Estimates 1992. Rockville (Maryland): Subtance Abuse and Mental Health Services Administration. USA. 1993.

8. OEDT. Observatorio Europeo de las Drogas y las Toxicomanías. Informe anual sobre el problema de las Drogodependencias en la Unión Europea. Lisboa 2004.

9. Plan nacional sobre drogas. Encuesta Domiciliaria sobre Uso de Drogas. Madrid. 1995-2005.

10. Plan nacional sobre drogas. Encuesta Escolar sobre el Uso de Drogas. Madrid. 1994-2006. 
11. OED. Observatorio Español sobre Drogas. Dirección General de Salud Pública. Ministerio de Sanidad. 2001-2007.

12. Navarro J. (Coord.) EDIS S.A. (Equipo de Investigación sociológica). La incidencia de las drogas en el mundo laboral de la comunidad de Madrid 2006. Observatorio Drogodependencias. Agencia Antidroga. Comunidad de Madrid. 2008.

13. Megías E, Navarro J. La incidencia de drogas en el mundo laboral. EDIS para FAD. Madrid 1996.

14. Fundación de Ayuda contra la Drogadicción, 2002. La incidencia de las drogas en el medio labora, 2001. Madrid FAD, 2001.

15. Navarro J, Rodríguez E. La incidencia de drogas en el mundo laboral. 2001. EDIS para FAD y PND. Madrid 2001.

16. ILO Home (International Labour Organization). Drug and alcohol abuse, an important workplace issue. www.ilo.org/public/english/protection/ safework/drug/ impiss. htm

17. Howland J, Almeida A, Rohsenow D, Minsky S, Greece J. How Safe are Federal Regulations on Occupational Alcohol Use? Journal Public Health Policy 2006.

18. Navarro J, Megías E. La incidencia de las drogas en el medio laboral de la Comunidad Valenciana. Fundación de Ayuda contra la Drogadicción y Generalitat Valenciana. Madrid 1999.

19. Pardo FJ. Predicción del consumo de drogas en el trabajo. Madrid 1996.

20. Mangione T, Howland J, Amick B, Cote J, Lee M, Bell N, et al. Employee drinking practices and work performance. J Stud Alcohol. 1999;60:261-70.

21. Salvador Llivina T, Suelves JM, del Pozo Irribarría J. Mano a mano. Programa para la prevención y asistencia de las drogodependencias en el medio laboral. Plan Riojano de Drogodependencias y otras Adicciones. 2003.

22. Rodríguez-Martos A, Navarro RM, Vecino C, Pérez R. Validación de los cuestionarios KFA (CBA) y CAGE para el diagnóstico del alcoholismo. Drogalcohol 1986; 11: 132-9.

23. Estatuto de los trabajadores. Real Decreto Legislativo 1/1995, de 24 de marzo, de la Ley del Estatuto de los Trabajadores.

24. Brodsky CM, Epstein IJ: The psychiatric disorders of aging in the work organization. Ocupational Medicine 1996; 11: 739-744.

25. Farid I, Brodsky CM. The management of psychopathology in the workplace. Occupational Medicine 1996; 11: 617-650.

26. Mingote JC, del Pino P, Huidobro A, Gutiérrez D, de Miguel I, Gálvez M. El paciente que padece un trastorno psicótico en el trabajo: diagnóstico y tratamiento. Medicina y Seguridad del Trabajo 2007; 2008: 29-52.

27. Ley $31 / 1995$, de 8 de noviembre, de Prevención de Riesgos Laborales. Artículo 22. www.boe.es/g/es/bases_datos/doc

28. Carrasco JJ, Maza JM. Manual de Psiquiatría legal y forense. Ed. La Ley. 2005. 